Accepted to ApJ Letters

Preprint typeset using $\mathrm{L}^{A} \mathrm{~T}_{\mathrm{E}} \mathrm{X}$ style emulateapj v. 05/12/14

\title{
THE KILOPARSEC-SCALE STAR FORMATION LAW AT REDSHIFT 4: WIDE-SPREAD, HIGHLY EFFICIENT STAR FORMATION IN THE DUST-OBSCURED STARBURST GALAXY GN20
}

\author{
J. A. Hodge ${ }^{1,2,3}$, D. Riechers ${ }^{4}$, R. Decarli ${ }^{2}$, F. Walter ${ }^{2}$, C. L. CArilli ${ }^{5,6}$, E. Daddi $^{7}$, And H. Dannerbauer $^{8}$ \\ Accepted to ApJ Letters
}

\begin{abstract}
We present high-resolution observations of the $880 \mu \mathrm{m}$ (rest-frame FIR) continuum emission in the $\mathrm{z}=4.05$ submillimeter galaxy GN20 from the IRAM Plateau de Bure Interferometer (PdBI). These data resolve the obscured star formation in this unlensed galaxy on scales of $0.3^{\prime \prime} \times 0.2^{\prime \prime}(\sim 2.1 \times 1.3 \mathrm{kpc})$. The observations reveal a bright $(16 \pm 1 \mathrm{mJy})$ dusty starburst centered on the cold molecular gas reservoir and showing a bar-like extension along the major axis. The striking anti-correlation with the HST/WFC3 imaging suggests that the copious dust surrounding the starburst heavily obscures the rest-frame UV/optical emission. A comparison with $1.2 \mathrm{~mm}$ PdBI continuum data reveals no evidence for variations in the dust properties across the source within the uncertainties, consistent with extended star formation, and the peak star formation rate surface density $\left(119 \pm 8 \mathrm{M}_{\odot} \mathrm{yr}^{-1} \mathrm{kpc}^{-2}\right)$ implies that the star formation in GN20 remains sub-Eddington on scales down to $3 \mathrm{kpc}^{2}$. We find that the star formation efficiency is highest in the central regions of GN20, leading to a resolved star formation law with a power law slope of $\Sigma_{\mathrm{SFR}} \sim \Sigma_{\mathrm{H}_{2}}^{2.1 \pm 1.0}$, and that GN20 lies above the sequence of normal star-forming disks, implying that the dispersion in the star formation law is not due solely to morphology or choice of conversion factor. These data extend previous evidence for a fixed star formation efficiency per free-fall time to include the star-forming medium on $\sim \mathrm{kpc}$-scales in a galaxy 12 Gyr ago.
\end{abstract}

Key words: galaxies: evolution - galaxies: formation - galaxies: high-redshift - galaxies: ISM galaxies: star formation

\section{INTRODUCTION}

One of the most commonly used diagnostics in studies of galaxy formation is the relation between gas surface density $\left(\Sigma_{\text {gas }}\right)$ and star formation rate (SFR) surface density $\left(\Sigma_{\mathrm{SFR}}\right)$, which describes the relative efficiency at which gas is transformed into stars in different environments (e.g. Schmidt 1959; Kennicutt 1998a). Recent studies indicate that this star formation (SF) law (or 'Kennicutt-Schmidt' law) is molecular (e.g., Bigiel et al. 2008; Lerov et al. 2008; Schruba et al. 2011), and that it does not evolve with redshift (e.g., Daddi et al. 2010; Genzel et al. 2010). There is evidence, however, for two different SF regimes on the $\Sigma_{\mathrm{SFR}}-\Sigma_{\mathrm{H},}$ plane ('main sequence galaxies' versus 'starbursts'; Daddi et al. 2010; Genzel et al. 2010, but c.f. Narayanan et al. 2012).

Due to the difficulty of resolving the cold molecular gas and obscured star-forming regions in distant star-forming galaxies, studies of the high-redshift SF law have historically been limited to unresolved de-

\footnotetext{
jhodge@nrao.edu

${ }^{1}$ National Radio Astronomy Observatory, 520 Edgemont Road, Charlottesville, VA, 22903, USA

${ }^{2}$ Max-Planck-Institut für Astronomie, Königstuhl 17, 69117 Heidelberg, Germany

3 Jansky Fellow

${ }^{4}$ Department of Astronomy, Cornell University, Ithaca, New York, 14853, USA

${ }^{5}$ National Radio Astronomy Observatory, P.O. Box 0, Socorro, NM 87801-0387, USA

${ }^{6}$ Astrophysics Group, Cavendish Laboratory, JJ Thomson Avenue, Cambridge CB3 0HE, UK

${ }^{7}$ CEA, Laboratoire AIM-CNRS-Université Paris Diderot, Irfu/SAp, Orme des Merisiers, F-91191 Gif-sur-Yvette, France

${ }^{8}$ Universität Wien, Institut für Astrophysik, Türkenschanzstraße 17, 1180 Wien, Austria
}

tections. However, it is clear that resolved observations are necessary to test whether the gas and SFR surface densities on sub-galactic scales are significantly different than those implied by global averages, as well as whether the most intensely star-forming galaxies at high-redshift - which appear to have $\Sigma_{\mathrm{SFR}}$ that are consistent with theories of so-called "maximum starbursts" (e.g. Elmegreen 1999; Tacconi et al. 2006) - are approaching the Eddington limit for dust in radiation pressure-supported disks (Scoville 2003; Thompson et al. 2005) on $\sim \mathrm{kpc}$, or even galaxy-wide scales (e.g., Walter et al. 2009; Riechers et al. 2013). While such resolved studies have become possible in the last few years, they are still limited to either $z<2$ (Freundlich et al. 2013) or gravitationally-lensed galaxies (Swinbank et al. 2010; Decarli et al. 2012; Sharon et al. 2013; Rawle et al. 2014).

To this end, we here report high-resolution rest-frame far-infrared (FIR) imaging of GN20 (Pope et al. 2006; Daddi et al. 2009), a $z=4.05$ submillimeter galaxy (SMG; Casey et al. 2014). GN20 has the deepest, highestresolution cold molecular gas imaging currently available for any unlensed high- $z$ galaxy, revealing an extended $(\sim 14 \pm 4 \mathrm{kpc})$ gas reservoir resolved on scales of $\sim 1.3$ kpc (Hodge et al. 2012, hereafter H12). The observations presented here provide nearly matched-resolution imaging of the dust emission, allowing us to investigate the SF efficiency (SFE) and SF law on $\sim \mathrm{kpc}$ scales 12 Gyr ago. Where applicable we assume a concordance, flat $\Lambda$ CDM cosmology $\left(\mathrm{H}_{0}=71 \mathrm{~km} \mathrm{~s}^{-1} \mathrm{Mpc}^{-1}, \Omega_{\Lambda}=0.73, \Omega_{M}=0.27\right.$; Spergel et al. 2007).

\section{OBSERVATIONS \& DATA REDUCTION}




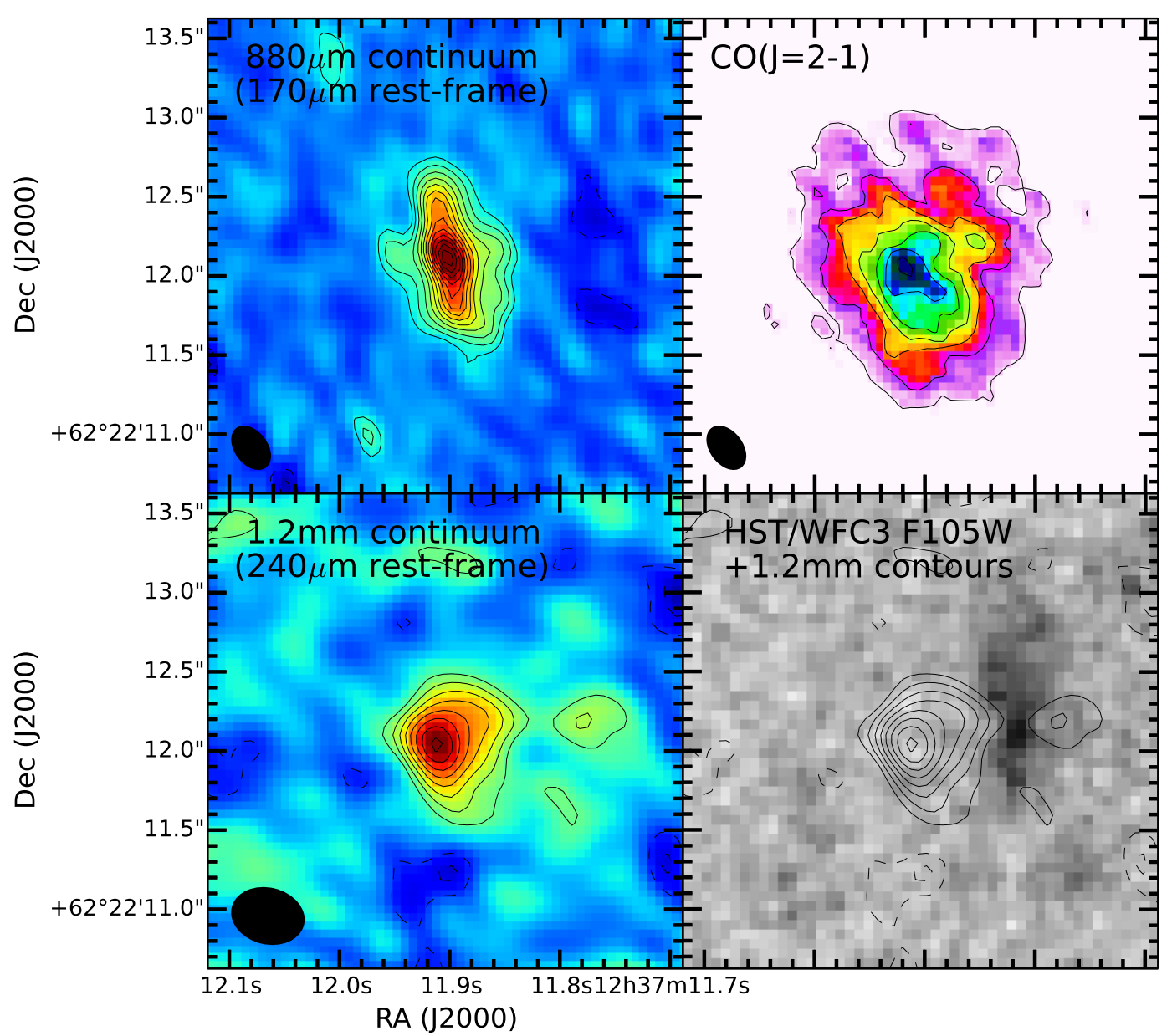

Figure 1. Multiwavelength imaging of the $\mathrm{z}=4.05$ SMG GN20. Top left: $880 \mu \mathrm{m}$ PdBI image $\left(0.3^{\prime \prime} \times 0.2^{\prime \prime}\right.$ resolution $)$. Contours start at $\pm 2 \sigma$ in steps of $1 \sigma=0.25 \mathrm{mJy}_{\text {beam }}{ }^{-1}$ (corresponding to a rest-frame brightness temperature of $\mathrm{T}_{B}=0.22 \mathrm{~K}$ ). The measured peak flux density of $3.6 \mathrm{mJy}$ implies $\mathrm{T}_{B, \text { peak }}=3.2 \mathrm{~K}$. Top right: VLA $\mathrm{CO}(2-1) 0$ th moment map (H12) at the same resolution as the $880 \mu \mathrm{m}$ data. Bottom left: $1.2 \mathrm{~mm}$ PdBI image $\left(0.46^{\prime \prime} \times 0.35^{\prime \prime}\right.$ resolution). Contours start at $\pm 2 \sigma$ in steps of $1 \sigma=0.25 \mathrm{mJy} \mathrm{beam}^{-1}$. Bottom right: HST/WFC3 F105W image from the CANDELS survey (Grogin et al. 2011) and 1.2mm contours.

\section{1. $P d B I 880 \mu m$}

The $880 \mu \mathrm{m}(170 \mu \mathrm{m}$ rest-frame) observations of GN20 $\left(\alpha(\mathrm{J} 2000)=12 \mathrm{~h} 37 \mathrm{~m} 11.920 \mathrm{~s}, \delta(\mathrm{J} 2000)=62^{\circ} 22^{\prime} 12.0^{\prime \prime}\right)$ were carried out in two tracks on 2013 December 4 (Cconfiguration track) and 2014 March 7 (A-configuration track) using six antennas. The observations used the PdBI's Band 4, tuned to $340 \mathrm{GHz}(880 \mu \mathrm{m})$, along with the WideX correlator $(3.6 \mathrm{GHz}$ bandwidth). The receiver was operating in the upper side band. The nearby radio quasars B1044+719 and B1418+546 were used for pointing, amplitude, and phase calibration, and the flux calibrators were 3C279 and MWC349 (A-track) and 3C84 and LKH $\alpha 101$ (C-track). We estimate the flux calibration to be good to within $20 \%$.

The IRAM GILDAS package was used for data reduction and analysis. The calibration process included two iterations of phase-only self-calibration, and one iteration of amplitude+phase self-calibration. After flagging, there were $5.7 \mathrm{~h} / 4.3 \mathrm{~h}$ on-source in the $\mathrm{C} / \mathrm{A}$-tracks (6-antenna equivalent). The final map (C+A tracks; Figure 1) has $0.05^{\prime \prime}$ pixels and was created using the CLEAN algorithm with robust weighting and a tight clean box on the source (cleaning down to $2 \sigma$ ). The image has a synthesized beam of $0.3^{\prime \prime} \times 0.2^{\prime \prime}$ and an $\mathrm{rms}$ of $0.25 \mathrm{mJy} \mathrm{beam}^{-1}$. As the source lies at the phase center of the $14.8^{\prime \prime}$ primary beam, the map was not corrected for primary beam attenuation.

\section{2. $P d B I$ 1.2mm}

The $1.2 \mathrm{~mm}(240 \mu \mathrm{m}$ rest-frame $)$ PdBI observations of GN20 (20.1" primary beam) were carried out in two tracks on 2010 February 1 and 7 using six antennas in the A-configuration. The $250 \mathrm{GHz}$ observations used the previous generation correlator, providing a total bandwidth of $1.0 \mathrm{GHz}$ (dual polarization). The quasars B1044+719, $\mathrm{B} 1418+546$, and B1300+580 were used for pointing, amplitude and phase calibration. Several standard calibrators (MWC349, Titan, 3C273, 3C279, 3C345, 3C454.3, $\mathrm{B} 0234+285, \mathrm{~B} 0923+392, \mathrm{~B} 1055+018, \mathrm{~B} 1749+096)$ were observed for flux and bandpass calibration, yielding $\sim 15 \%$ calibration accuracy.

The data were mapped using CLEAN with natural weighting, resulting in a synthesized beam of $0.46^{\prime \prime} \times 0.35^{\prime \prime}$ (Figure 1). Weighting schemes that would lead to higher spatial resolution were discarded due to high noise. The final $\mathrm{rms}$ noise is $0.25 \mathrm{mJy} \mathrm{beam}^{-1}$ over 


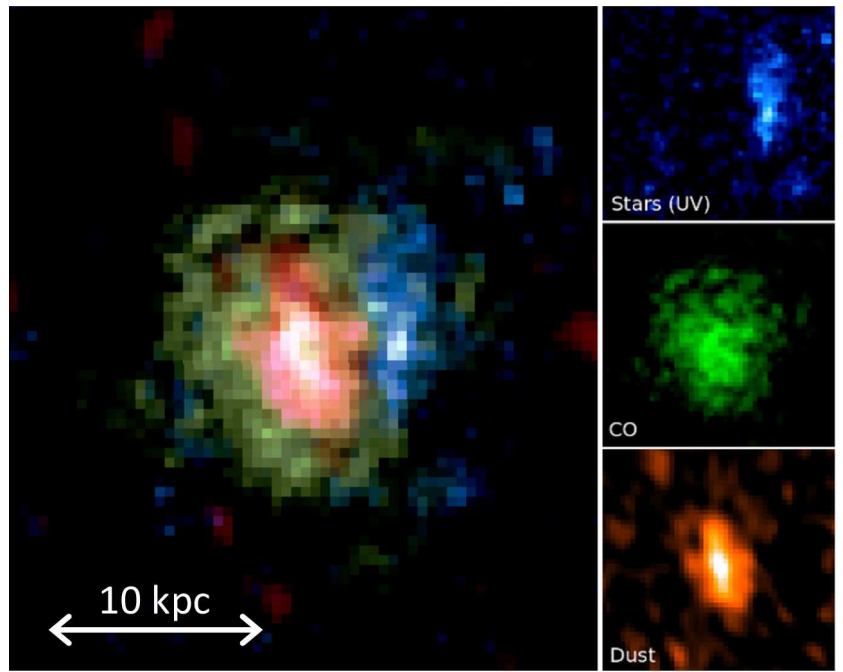

Figure 2. False-color image of GN20, showing the HST/WFC3 F105W emission (tracing young stars; blue), the VLA CO $(2-1)$ emission (tracing cold molecular gas; green), and the PdBI $880 \mu \mathrm{m}$ emission (tracing dust-obscured SF; red).

the full bandpass.

\subsection{VLA $C O(2-1)$}

The $\mathrm{CO}(2-1)$ data on GN20 were obtained with the Karl G. Jansky Very Large Array (VLA) and presented in Carilli et al. (2011) and H12. The 0th moment map used here (Figure 11) was created by taking the native resolution $\left(0.19^{\prime \prime} / 1.3 \mathrm{kpc}\right)$ data cube and convolving it to the resolution of the $880 \mu \mathrm{m}$ data $\left(0.3^{\prime \prime} \times 0.2^{\prime \prime}\right)$, applying the same mask as used in H12.

\section{RESULTS}

\subsection{Spatially-resolved rest-frame FIR emission}

We have detected and spatially-resolved the $880 \mu \mathrm{m} \quad(170 \mu \mathrm{m}$ rest-frame $)$ continuum emission from GN20 (Figure 11). We measure a peak flux density of $3.6 \mathrm{mJy}$ beam $^{-1}$ at $\alpha(\mathrm{J} 2000)=12 \mathrm{~h} 37 \mathrm{~m} 11.90 \mathrm{~s}$, $\delta(\mathrm{J} 2000)=62^{\circ} 22^{\prime} 12.10^{\prime \prime}$, positionally coincident with the peak of the CO emission within $0.1^{\prime \prime}$, and suggesting that the most intense SF occurs in the region with the highest concentration of cold gas. The integrated flux density $(16 \pm 1 \mathrm{mJy})$ is consistent with the integrated flux density measured in the C-configuration data alone $\left(18 \mathrm{mJy}\right.$; beam $\left.0.88^{\prime \prime} \times 0.74^{\prime \prime}\right)$, indicating that the higher-resolution data are not resolving out a significant amount of emission. These measurements are also consistent with the single dish measurement for GN20 $\left(\mathrm{S}_{850}=20.3 \pm 2.1 \mathrm{mJy}\right.$; Pope et al. 2006). Note that despite its large flux density, the evidence suggests that GN20 is not lensed (Carilli et al. 2010).

The full size of GN20's molecular gas reservoir is $14 \pm 4 \mathrm{kpc}$ (H12). From the new $880 \mu \mathrm{m}$ continuum data, we find that the dust-obscured SF is also extended, with a bar-like extension along the major axis of the galaxy. We measure a deconvolved source size of $\left(0.76^{\prime \prime} \pm 0.06^{\prime \prime}\right) \times\left(0.33^{\prime \prime} \pm 0.03^{\prime \prime}\right)(\mathrm{FWHM}$, equivalent to $\sim 5.3 \times 2.3 \mathrm{kpc}$ ), implying a physical size of $\sim 10 \mathrm{kpc}$ out to $10 \%$ of the peak flux density (along its major axis). This suggests that the dust-obscured SF in this galaxy is almost as extended as the gas reservoir - a conclusion

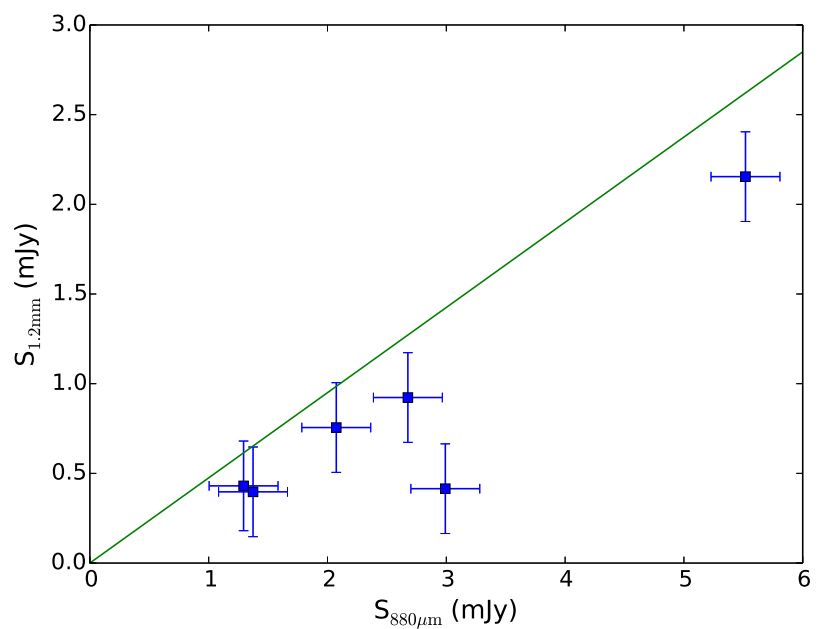

Figure 3. $\mathrm{S}_{880 \mu \mathrm{m}}$ vs. $\mathrm{S}_{1.2 \mathrm{~mm}}$ for $0.4^{\prime \prime}(\sim 2.8 \mathrm{kpc})$ resolution elements. The solid line represents the ratio expected from the best-fit model to GN20's IR SED (Tan et al. 2014).

which is consistent with the spatially-resolved excitation structure (H12).

While the FIR emission is coincident with the CO emission, both the FIR and $\mathrm{CO}$ emission are offset by $\sim 0.6^{\prime \prime}$ (4 kpc) from the peak of the rest-frame UV emission as traced by the HST/WFC3 F105W image (Figure 21). The striking anti-correlation suggests that the copious dust surrounding the starburst heavily obscures the UV/optical light produced by newly-formed stars and keeps it from escaping the galaxy in all but one small region several kpc from the nuclear burst.

We have also detected and resolved GN20 in the $1.2 \mathrm{~mm} \quad(240 \mu \mathrm{m}$ rest-frame $)$ continuum observations (Figure 11). We measure an integrated flux density of $5.7 \pm 1.6 \mathrm{mJy}$, which is $\sim 2 / 3$ of the flux measured with the MAMBO- 2 bolometer at $11^{\prime \prime}$ resolution at the same wavelength (9.3 $\pm 0.9 \mathrm{mJy}$; Greve et al. 2008). As the $1.2 \mathrm{~mm}$ data were taken entirely in the extended configuration, this could indicate that some spatially-extended flux on $\geq 1^{\prime \prime}$ scales is resolved out. The FWHM source size of $\left(0.77^{\prime \prime} \pm 0.17^{\prime \prime}\right) \times\left(0.49^{\prime \prime} \pm 0.17^{\prime \prime}\right)$ measured from the $1.2 \mathrm{~mm}$ observations is consistent with that measured from the $880 \mu \mathrm{m}$ data.

\subsection{Dust continuum slope}

We used our dust imaging to examine possible deviations from a constant $880 \mu \mathrm{m} / 1.2 \mathrm{~mm}$ ratio, which could suggest variations in the dust continuum slope across the galaxy. A gradient in the slope could indicate a gradient in the dust temperature and/or optical depth - both of which may be expected for dense nuclear starbursts. We convolved the $880 \mu \mathrm{m}$ image to the resolution of the $1.2 \mathrm{~mm}$ image, resampled both maps to have $0.4^{\prime \prime}$ pixels $(\sim 2.8 \mathrm{kpc}$, the approximate size of the resolution element), and blanked all pixels except those with flux densities $>1 \sigma$ in both maps in a central contiguous region. The resulting flux densities are plotted in Figure3. where the solid line represents the ratio expected from the bestfit Draine \& Li (2007) dust model to the IR SED - see Tan et al. (2014, hereafter T14) for further details. In general, we find $1.2 \mathrm{~mm}$ flux densities that are $\sim 20-25 \%$ 

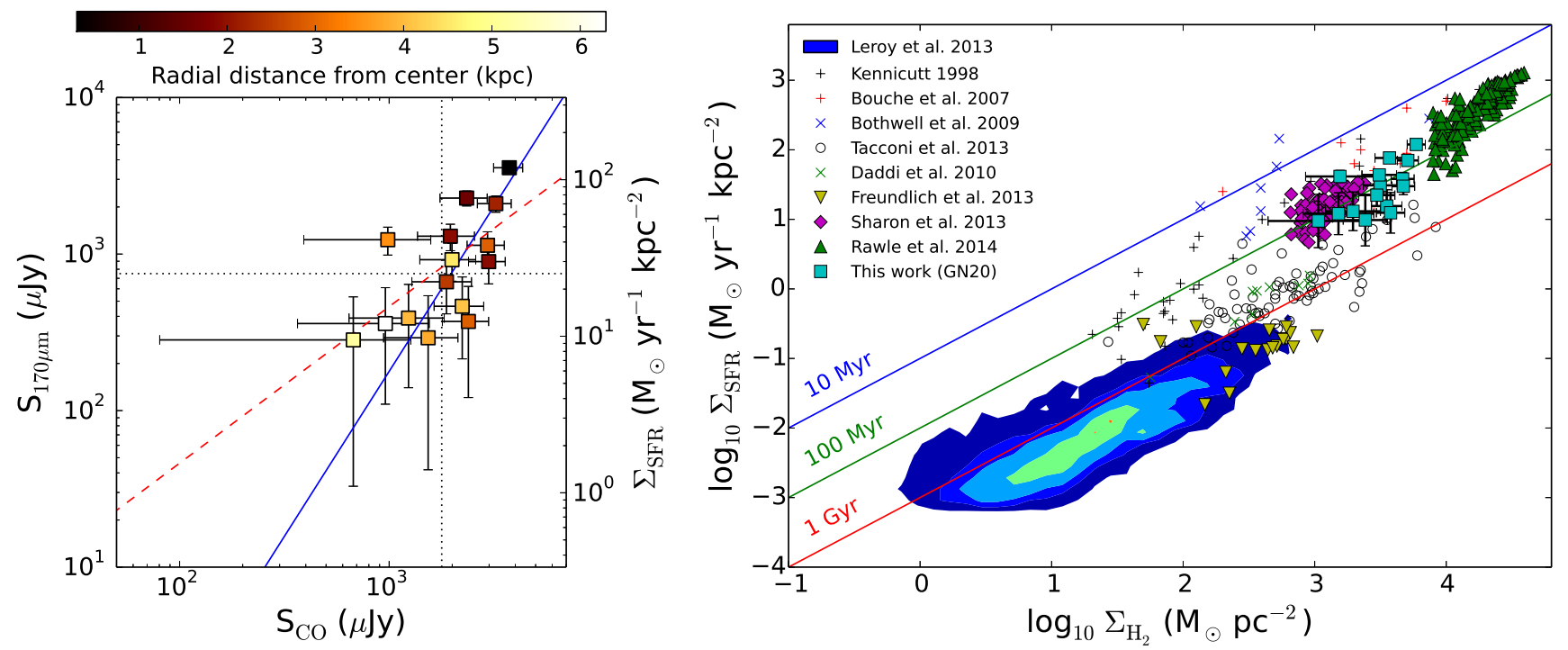

Figure 4. The relation between molecular gas and SF in GN20. Left: Rest-frame $170 \mu \mathrm{m}$ flux density vs. CO(2-1) flux density, colorcoded by distance from the galactic center. The right-hand axis shows $\Sigma_{\mathrm{SFR}}$ (see Section 3.3). The solid blue line shows a power law fit to the data, including points below $3 \sigma$ (indicated by black dotted lines), and the red dashed line shows a slope of unity (indicating constant SFE). Right: $\Sigma_{\mathrm{SFR}}$ vs. $\Sigma_{\mathrm{H}_{2}}$ in GN20 and other local and high-redshift sources from the literature. Contours indicate the density of local galaxies (taken from Lerov et al. 2013). Unfilled data points show unresolved measurements, including local ULIRGs (black plus-signs; Kennicutt $1998 \mathrm{~b}$ ); $\mathrm{z} \sim 0.5$ disk galaxies and z 1.5 BzK galaxies (green crosses; Daddi et al. 2010); z 1-3 color-selected galaxies (black circles; Tacconi et al.|2010); and SMGs as red plus-signs (Bouché et al. 2007) and blue crosses (Bothwell et al.|2010). Filled symbols show resolved measurements, including z 1.2 massive star-forming galaxies (yellow upside-down triangles; Freundlich et al. 2013); two strongly-lensed SMGs as magenta diamonds (Sharon et al.|2013) and green triangles (Rawle et al.|2014); and this work on GN20 (cyan squares). The solid lines indicate constant gas depletion timescales of $10 \mathrm{Myr}$ (blue), $100 \mathrm{Myr}$ (green), and $1 \mathrm{Gyr}$ (red).

lower than expected, providing further evidence that the lack of short baseline information may have resolved out some extended emission 9 The remaining scatter, however, is not statistically significant. We conclude that the data are consistent with a constant ratio, with no evidence for a variation in the dust continuum slope across the galaxy. This finding is consistent with the apparently extended nature of the SF in GN20, and it supports the use of a constant $\Sigma_{\mathrm{SFR}} / \mathrm{S}_{880 \mu m}$ conversion factor in the analysis that follows.

\subsection{SFR surface densities}

GN20 has an infrared luminosity of $\log \left(L_{\mathrm{IR}} / \mathrm{L}_{\odot}\right)=13.27 \pm 0.02$, implying a total SFR of $1860 \pm 90 \mathrm{M}_{\odot} \mathrm{yr}^{-1}$ (assuming a Chabrier IMF; T14). This is consistent with an analysis of the PAH emission (Riechers et al. 2014), indicating no significant AGN contamination to $L_{\mathrm{IR}}$. Using the extent of the $880 \mu \mathrm{m}$ emission, the average $\Sigma_{\mathrm{SFR}}$ is $\sim 100 \mathrm{M}_{\odot} \mathrm{yr}^{-1} \mathrm{kpc}^{-2}$. To determine how $\Sigma_{\mathrm{SFR}}$ varies on $\sim \mathrm{kpc}$ scales, we resampled the $880 \mu \mathrm{m}$ map onto a grid of $0.25^{\prime \prime} / 1.75 \mathrm{kpc}$ pixels and used the best-fit Draine \& Li (2007) dust model to the IR SED (T14) to determine a conversion factor between the $880 \mu \mathrm{m}$ flux densities and IR luminosities of $1.006( \pm 0.045) \times 10^{12} \mathrm{~L}_{\odot} \mathrm{mJy}^{-1}$. The uncertainty derives from the uncertainty on the (model-derived) $L_{\mathrm{IR}}$, which was quantified by T14 using a series of $\mathrm{MC}$ simulations. We then divided by the beam area and scaled based on the total SFR/ $L_{\mathrm{IR}}$ for GN20. We

\footnotetext{
${ }^{9}$ Another possibility is that the $880 \mu \mathrm{m}$ flux density is boosted by the presence of $\mathrm{H}_{2} 0\left(3_{03}-2_{12}\right)$ emission $\left(\nu_{\text {rest }}=1716.769633 \mathrm{GHz}\right)$, but taking its $3 \sigma$ upper limit (given its non-detection), and assuming the $\mathrm{CO}(2-1)$ linewidth $\left(730 \mathrm{~km} \mathrm{~s}^{-1}\right)$, we limit its contribution to $\lesssim 3.5 \%$ of the measured continuum flux.
}

find values in the $\sim$ tens of $\mathrm{M}_{\odot} \mathrm{yr}^{-1} \mathrm{kpc}^{-2}$, peaking at $119 \pm 8 \mathrm{M}_{\odot} \mathrm{yr}^{-1} \mathrm{kpc}^{-2}$ in the galaxy's center.

\subsection{The resolved $S F$ law at $z=4$}

In order to study the spatially-resolved SF law in GN20, we resampled the $\mathrm{CO}(2-1)$ map onto the pixel grid described in Section 3.3. The observed flux densities from the $\mathrm{CO}$ and $880 \mu \mathrm{m}$ maps are plotted in Figure 4 (left). The ratio between FIR and CO emission (indicating SFE, and thus gas depletion timescale) is highest in the central regions of the galaxy, which have the highest gas columns. The SFE tends to decrease further from the galactic center, with an overall variation of $\sim 8$ across the regions where most of the SF takes place. A function of the form $\Sigma_{880 \mu \mathrm{m}} \sim\left(\Sigma_{\mathrm{CO}}\right)^{\mathrm{N}}$ was fit to the data using the IDL routine LINMIX_ERR (Kelly 2007), a linear regression estimator that uses a Bayesian approach and considers measurement errors in two variables. The result of the fit gives a slope of $\mathrm{N}=2.1 \pm 1.0$. This slope assumes a constant $\Sigma_{\mathrm{SFR}} / \mathrm{S}_{880 \mu \mathrm{m}}$ ratio, as supported by our analysis in Section 3.2. To simulate the effect of a variable dust temperature across the source, we re-ran the Draine \& Li models with a wide range of ionization parameters $\left(\mathrm{U}_{\min }=10-100\right)$, resulting in $\Sigma_{\mathrm{SFR}}$ increasing/decreasing by less than a factor of two. Given the radial trend in SFE, this would increase the vertical scatter in the data, steepening the slope.

Figure 4 (right) shows the GN20 data on the $\Sigma_{\mathrm{SFR}}-\Sigma_{\mathrm{H}_{2}}$ plane. In order to convert the $\mathrm{CO}(2-1)$ flux densities to molecular gas masses, we have assumed the standard relationships from Carilli \& Walter (2013) and a CO-to$\mathrm{H}_{2}$ conversion factor of $\alpha_{\mathrm{CO}}=1.1 \mathrm{M}_{\odot}\left(\mathrm{K} \mathrm{km} \mathrm{s}^{-1} \mathrm{pc}^{2}\right)^{-1}$. This value - which is slightly higher than the typicallyadopted ULIRG value - was determined in H12 by using the dynamical mass (derived by modeling the $\mathrm{CO}(2-1)$ 


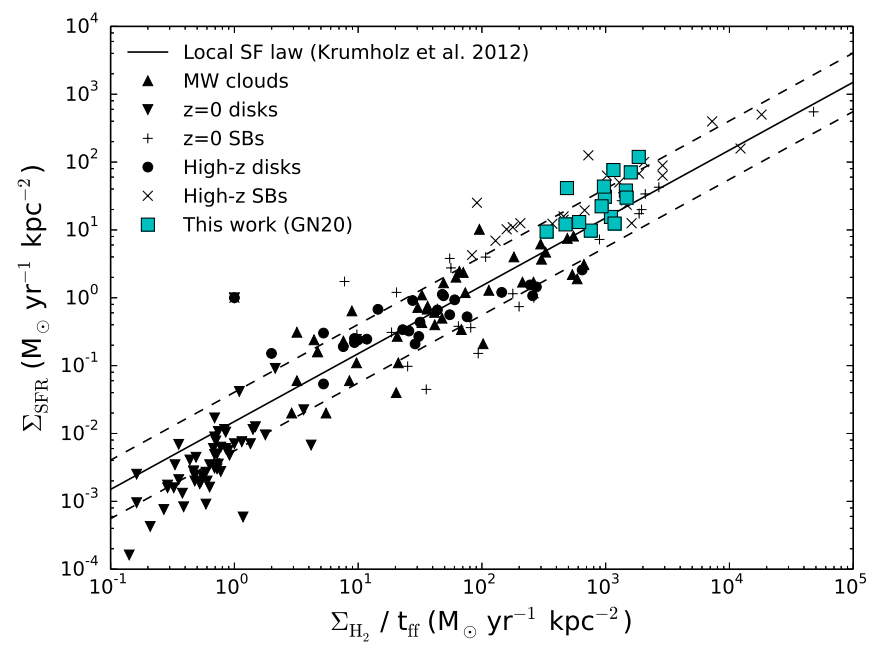

Figure 5. $\Sigma_{\mathrm{SFR}}$ vs. $\Sigma_{\mathrm{H}_{2}} / \mathrm{t}_{\mathrm{ff}}$ for GN20 (cyan squares) and a variety of comparison sources (Krumholz et al. 2012). The black solid (dashed) lines show the local volumetric SF law (and scatter) from Krumholz et al. (2012), which assumes their best-fit value of $\epsilon_{\mathrm{ff}}=0.015$.

dynamics) to constrain the gas content10. The mean depletion time we measure for GN20 is $130 \mathrm{Myr}$, with a range of 40-300 Myr. A compilation of literature studies is shown for comparison, where all SFR measurements have been converted to a common Chabrier (2003) IMF. The gas masses were calculated using the CO-to$\mathrm{H}_{2}$ conversion factors assumed in the respective studies: $\alpha_{\mathrm{CO}}=0.8$ for the unresolved ULIRGs/SMGs (including Helium; Kennicutt 1998b; Bouché et al. 2007; Bothwell et al. 2010); $\alpha_{\mathrm{CO}}=3.6$ for the $\mathrm{z} \sim 0.5$ disk galaxies and BzKs (Daddi et al. 2010); $\alpha_{\mathrm{CO}}=4.35$ for the local spirals and color-selected galaxies (Tacconi et al. 2010; Lerov et al. 2013; Freundlich et al. 2013); and $\alpha_{\mathrm{CO}}=4.6$ (Sharon et al. 2013) and $\alpha_{\mathrm{CO}}=0.7$ (Rawle et al. 2014) for the two strongly-lensed resolved SMG:11, 12.

Figure 5 shows $\Sigma_{\mathrm{SFR}}$ versus $\Sigma_{\mathrm{H}_{2}}$, where the latter is divided by free-fall time $\left(t_{\mathrm{ff}}\right)$. The comparison data are from Krumholz et al. (2012). To calculate $t_{f f}$ for GN20, we used equation 8 from Krumholz et al., which is appropriate for high surface density galaxies in the Toomre regime, and we have assumed the Toomre parameter $\mathrm{Q}=1$, the dimensionless constant $\phi_{P}=3$ (Krumholz \& McKee 2005), the logarithmic index of the rotation curve $\beta=0$ (flat rotation curve; H12), and the angular velocity of galactic rotation $\Omega=0.175 \mathrm{Myr}^{-1}$ (calculated at the half-light radius). For GN20, this gives values of the SFE per free-fall time $\left(\epsilon_{\mathrm{ff}}\right.$; i.e., the fraction of gas converted into stars per free-fall time) ranging from $\epsilon_{\mathrm{ff}}=0.01-0.18$. Also shown is the local volumetric SF law from Krumholz et al. (2012), evaluated at their best-fit value of $\epsilon_{\mathrm{ff}}=0.015$.

\section{DISCUSSION}

\footnotetext{
10 As $\alpha_{\mathrm{CO}}$ was dynamically-constrained directly from $\mathrm{CO}(2-1)$, there is no need to assume a $\mathrm{CO}(2-1) / \mathrm{CO}(1-0)$ excitation ratio.

${ }^{11}$ Units same as above.

12 Note that the Rawle et al. and Sharon et al. data are oversampled - see their papers for details.
}

While it is evident that SMGs host some of the largest starbursts in the known Universe $\left(\sim 10^{3} \mathrm{M}_{\odot} \mathrm{yr}^{-1}\right)$, this knowledge is largely based on integrated measurements and/or indirect SFR tracers. The latter imply that SMGs have typical SFR densities of $\sim 80 \mathrm{M}_{\odot} \mathrm{yr}^{-1} \mathrm{kpc}^{-2}$ (Tacconi et al. 2006), well below the $\sim 1000 \mathrm{M}_{\odot} \mathrm{yr}^{-1} \mathrm{kpc}^{-2}$ limit based on a theoretical description of the (dust-opacity) Eddington-limited SF of a radiation pressure supported starburst on $\mathrm{kpc}$ scales (Scoville 2003; Thompson et al. 2005), though exceptions do exist (e.g., $\sim 600 \mathrm{M}_{\odot} \mathrm{yr}^{-1} \mathrm{kpc}^{-2}$ in the $\mathrm{z}=6.3$ SMG HFLS3; Riechers et al. 2013). Indeed, the highest-resolution existing submillimeter observations on the lensed $\mathrm{z} \sim 2.3$ "Eyelash" galaxy show that the star-forming regions are undergoing maximal starbursts (Swinbank et al. 2010). Previous $0.8^{\prime \prime} 890 \mu \mathrm{m}$ imaging (Younger et al. 2008) of GN20 - which is intrinsically much more luminous than the Eyelash - indicated that it might be forming stars close to or at its Eddington limit, although subsequent CO imaging (Carilli et al. 2010) revealed the large gas disk and, along with a revised total SFR (Daddi et al. 2009), implied a sub-Eddington average value. Still, it was unknown whether the SF in GN20 was more compact than the cold gas reservoir traced by $\mathrm{CO}(2-1)$, or whether the indirectly-derived average value served to mask extreme differences in $\Sigma_{\mathrm{SFR}}$ between clump/inter-clump regions. Our high-resolution $880 \mu \mathrm{m}$ imaging shows that the SF in GN20 remains subEddington on scales down to a few $\mathrm{kpc}^{2}$.

An examination of the resolved SF law in this unlensed $\mathrm{z} \sim 4$ galaxy produced a power-law slope of $\mathrm{N}=2.1 \pm 1.0$. While the significant uncertainty on the slope means that it may be only barely steeper than the local linear relation (e.g., Leroy et al. 2013), other studies also find evidence for a steepening in gas-rich/high density environments (i.e. $\geq 200 \mathrm{M}_{\odot}$ pc $^{-2}$; Solomon et al. 1987; Gao \& Solomon 2004; Rosolowsky \& Blitz 2005), including the centers of nearby galaxies (e.g., Narayanan et al. 2008; Leroy et al. 2013). A slope of $N \sim 1.5$ may be expected if SFR is proportional to gas mass divided by free-fall time (assuming a constant scale height; e.g., Madore 1977; Krumholz et al. 2009). Other models of feedback-regulated SF (e.g. Ostriker \& Shetty 2011; Faucher-Giguère et al. 2013) give a quadratic slope closer to that seen here, though it is impossible to differentiate between models given the current observational uncertainties.

When compared with other studies on the $\Sigma_{\mathrm{SFR}}-\Sigma_{\mathrm{H}_{2}}$ plane, we find that the GN20 data lie above the sequence traced out by 'normal' star-forming disk galaxies. This is consistent with its classification as a starburst (T14) and, given that the $\mathrm{CO}(2-1)$ data show evidence for a smoothly rotating gas disk (H12), it implies that the presence of an ordered velocity field is not a clear indication of the presence or absence of intense starburst activity. The approximate alignment of GN20 and the two (resolved) lensed SMGs is also notable. Given that the conversion factors specifically-derived for the individual cases range from ULIRG-like (Rawle et al. 2014) to slightly above ULIRG-like (GN20) to Galactic (Sharon et al. 2013), this suggests that the dispersion in the SF law is apparently not due solely to choice of $\alpha_{\mathrm{CO}}$. On the contrary, the GN20 results suggest that the dis- 
persion in the SF law is real, and that SMGs can have very high star formation efficiencies on small scales.

If one assumes a universal SF law where the SFR volume density is simply a function of the molecular gas volume density and local $t_{f f}$ (Schmidt 1959; Kennicutt 1998b; Krumholz \& McKee 2005; Leroy et al. 2008), then the complexity in the $\Sigma_{\mathrm{SFR}}-\Sigma_{\mathrm{H}_{2}}$ plane should be removed by accounting for $t_{f f}$. Studies substituting the global dynamical timescale $\left(t_{d y n}\right)$ for $t_{f f}$ in disk galaxies and mergers at low/high-redshift (Daddi et al. 2010; Genzel et al. 2010) provided preliminary support for this theory, as $t_{d y n}$ is proportional to $t_{f f}$ for galactic scales in high surface density galaxies (Krumholz \& McKee 2005; Lerov et al. 2008; Genzel et al. 2010; Krumholz et al. 2012). More recently, Krumholz et al. (2012) presented a complete theoretical framework for a local volumetric SF law, including a model for the local (star-forming cloud) $t_{\mathrm{ff}}$, which allowed them to describe the SF on scales from entire high-redshift galaxies down to individual molecular clouds with a fixed value of $\epsilon_{\mathrm{ff}}=0.015$. Figure 5 demonstrates that our resolved imaging of GN20 is also consistent with this model, extending the evidence for a fixed SFE per free-fall time to include the star-forming medium on $\sim$ kpc-scales in a galaxy 12 Gyr ago.

We thank John Hibbard, Mark Krumholz, Adam Leroy, Kartik Sheth, and an anonymous referee for helpful comments/discussions, and Roberto Neri, Sabine König, and Melanie Krips for help with data analysis. IRAM is supported by INSU/CNRS (France), MPG (Germany) and IGN (Spain).

\section{REFERENCES}

Bigiel, F., Leroy, A., Walter, F., et al. 2008, AJ, 136, 2846 Bothwell, M. S. et al. 2010, MNRAS, 405, 219

Bouché, N. et al. 2007, ApJ, 671, 303

Carilli, C. L., Daddi, E., Riechers, D., et al. 2010, ApJ, 714, 1407

Carilli, C. L., Hodge, J., Walter, F., et al. 2011, ApJ, 739, L33

Carilli, C. L. \& Walter, F. 2013, ARA\&A, 51, 105

Casey, C. M., Narayanan, D., \& Cooray, A. 2014, ArXiv e-prints Chabrier, G. 2003, ApJ, 586, L133

Daddi, E., Dannerbauer, H., Stern, D., et al. 2009, ApJ, 694, 1517

Daddi, E., Elbaz, D., Walter, F., et al. 2010, ApJ, 714, L118

Decarli, R., Walter, F., Neri, R., et al. 2012, ApJ, 752, 2
Draine, B. T. \& Li, A. 2007, ApJ, 657, 810

Elmegreen, B. G. 1999, ApJ, 517, 103

Faucher-Giguère, C.-A., Quataert, E., \& Hopkins, P. F. 2013, MNRAS, 433, 1970

Freundlich, J., Combes, F., Tacconi, L. J., et al. 2013, A\&A, 553, A130

Gao, Y. \& Solomon, P. M. 2004, ApJ, 606, 271

Genzel, R., Tacconi, L. J., Gracia-Carpio, J., et al. 2010, MNRAS, 407, 2091

Greve, T. R., Pope, A., Scott, D., et al. 2008, MNRAS, 389, 1489

Grogin, N. A., Kocevski, D. D., Faber, S. M., et al. 2011, ApJS, 197,35

Hodge, J. A., Carilli, C. L., Walter, F., et al. 2012, ApJ, 760, 11

Kelly, B. C. 2007, ApJ, 665, 1489

Kennicutt, Jr., R. C. 1998a, ARA\&A, 36, 189

-. 1998b, ApJ, 498, 541

Krumholz, M. R., Dekel, A., \& McKee, C. F. 2012, ApJ, 745, 69

Krumholz, M. R. \& McKee, C. F. 2005, ApJ, 630, 250

Krumholz, M. R., McKee, C. F., \& Tumlinson, J. 2009, ApJ, 699, 850

Leroy, A. K., Walter, F., Brinks, E., et al. 2008, AJ, 136, 2782

Leroy, A. K., Walter, F., Sandstrom, K., et al. 2013, AJ, 146, 19

Madore, B. F. 1977, MNRAS, 178, 1

Narayanan, D., Cox, T. J., \& Hernquist, L. 2008, ApJ, 681, L77

Narayanan, D., Krumholz, M. R., Ostriker, E. C., \& Hernquist, L. 2012, MNRAS, 421, 3127

Ostriker, E. C. \& Shetty, R. 2011, ApJ, 731, 41

Pope, A., Scott, D., Dickinson, M., et al. 2006, MNRAS, 370, 1185

Rawle, T. D., Egami, E., Bussmann, R. S., et al. 2014, ApJ, 783, 59

Riechers, D. A., Pope, A., Daddi, E., et al. 2014, ApJ, 786, 31

Riechers, D. A. et al. 2013, Nature, 496, 329

Rosolowsky, E. \& Blitz, L. 2005, ApJ, 623, 826

Schmidt, M. 1959, ApJ, 129, 243

Schruba, A., Leroy, A. K., Walter, F., et al. 2011, AJ, 142, 37

Scoville, N. 2003, Journal of Korean Astronomical Society, 36, 167

Sharon, C. E., Baker, A. J., Harris, A. I., \& Thomson, A. P. 2013, ApJ, 765, 6

Solomon, P. M., Rivolo, A. R., Barrett, J., \& Yahil, A. 1987, ApJ, 319, 730

Spergel, D. N. et al. 2007, ApJS, 170, 377

Swinbank, A. M. et al. 2010, Nature, 464, 733

Tacconi, L. J., Neri, R., Chapman, S. C., et al. 2006, ApJ, 640, 228

Tacconi, L. J. et al. 2010, Nature, 463, 781

Tan, Q., Daddi, E., Magdis, G., et al. 2014, A\&A, 569, A98

Thompson, T. A., Quataert, E., \& Murray, N. 2005, ApJ, 630, 167

Walter, F., Riechers, D., Cox, P., et al. 2009, Nature, 457, 699

Younger, J. D. et al. 2008, ApJ, 688, 59 